\title{
Modeling and Visualizing Uncertainty in a Global Water Balance Model
}

\author{
James R. Miller \\ University of Kansas \\ 1460 Jayhavk Blvd \\ Lawrence, KS 66045 \\ 785-864-7384 \\ jmiler@ku.edu
}

\author{
Daniel C. Clib um \\ Hanover College \\ P. O. Box 890 \\ Hanover, IN 47243 \\ 812-866-7288 \\ cliburn@hanover.edu
}

\author{
Johames J. Feddema \\ University of Kansas \\ 1475 Jayhawk Blvd \\ Lawrence, KS 66045 \\ 785-864-5534 \\ feddema@ku.edu
}

\author{
Terry A. Slocum \\ University of Kansas \\ 1475 Jayhawk Blvd \\ Lawrence, KS 66045 \\ 785-864-5538 \\ t-slocum@ku.edu
}

\begin{abstract}
Public policy makers and others wishing to understand the potential impact of our actions on the environment need a tool to simulate and visualize relevant processes. Present models and the data on which they operate have considerable variability which translates into uncertainty in model results. Decision makers must understand and consider not only raw model results, but also the uncertainties associated with them. As an example, we describe a tool that evaluates a global water balance model and presents visualizations of both the results and the associated uncertainties.
\end{abstract}

\section{Keywords}

Visualization, uncertainty, collaboration, decision support

\section{INTRODUCTION}

As our understanding of natural phenomena has improved in recent times, we have become more reliant on computer simulations for decision making. Although simulations can often provide useful information, even in the best environmental modeling and simulation efforts there remain knowledge gaps and various types of uncertainties associated with data collection and manipulation, model accuracy, and people's interpretation of data and model results. A good example of such uncertainties is demonstrated in simulations of global climate change. Uncertainty in data and model results have had a profound impact on the interpretation of climate modeling results, ultimately resulting in stark international political conflicts as seen in the results of the Rio and Kyoto global climate change summit meetings.

A part of the problem is the fact that sophisticated computer graphics approaches allow us to produce attractive high-quality 3D color images that impart "truth" to simulations. In reality, however, simulations are characterized by uncertainty, both in the data and in the

Permission to make digital or hard copies of all or part of this work for personal or classroom use is granted without fee provided that copies are not made or distributed for profit or commercial advantage and that copies bear this notice and the full citation on the first page. To copy otherwise, or republish, to post on servers or to redistribute to lists, requires prior specific permission and/or a fee.

SAC 2003, March 9-12, 2003, Melbourne, Florida, USA.

Copyright 2003 ACM 1-58113-624-2/03/03 ...\$5.00. model used to create the simulations. When designing visualization tools to be used in a decision support context for policy related to natural phenomena, it is incumbent upon the system designer to display not only results of simulations, but also a reasonable estimate of the uncertainty associated with those results. That is, decision makers must understand not only the results, but also the reliability of those results.

In this paper, we will use a global water balance model and a suite of visualization tools to illustrate some approaches for visualizing the uncertainty associated with simulations of natural phenomena and global climate change scenarios. By clearly presenting the relative impacts of several different modeling and data factors on model results, we hope to improve decision makers' ability to better understand the nature and causes of uncertainty in climate prediction. The resulting system is intended to be a collaborative visualization tool that will allow domain experts and policy makers to work together to explore the effects of potential land use decisions.

\section{WATER BUDGETS AND GLOBAL CLIMATE CHANGE}

When addressing future climate change, the potential outcomes of human activities on climate can only be simulated through the use of Global Circulation Models (GCMs). Yet most political decision makers have relatively little knowledge of the scientific processes involved in such models and the associated uncertainties involved in interpreting the results. Some types of uncertainties are more problematic than others. For example, if the input data has errors, but these errors propagate systematically through the model and consistently through all experiments, such error is not considered to have a significant impact on the interpretation of results. However, errors or uncertainties that do not propagate systematically through the system, such as choices of different sub-model components, may have a greater impact on uncertainty with respect to decision making. We are most interested in these latter sorts of uncertainties.

We have chosen a water balance model to illustrate the use of uncertainty presentations to assist decision-makers in making more informed decisions about the potential impacts of climate change. Water is one of the most critical resources to modern societies. Changes in the 
allocation and distribution of water resources can have significant effects on local economies and agricultural production. Therefore, a basic understanding of the changes in this resource will provide valuable information on the potential economic and social impacts of climate change.

The water balance model used in this study is based on a water accounting procedure first developed by Thornthwaite [24]. The model tracks water movement through an environment and is based on evaluating the change in the soil moisture content for a location. Water gains come from precipitation, while water losses come from overland runoff and through evaporation and transpiration. From the model, it is possible to detect water deficit and water surplus conditions throughout the year (Figure 1). The current configuration of the model only requires precipitation, temperature, latitude, and soil water holding capacity as input.

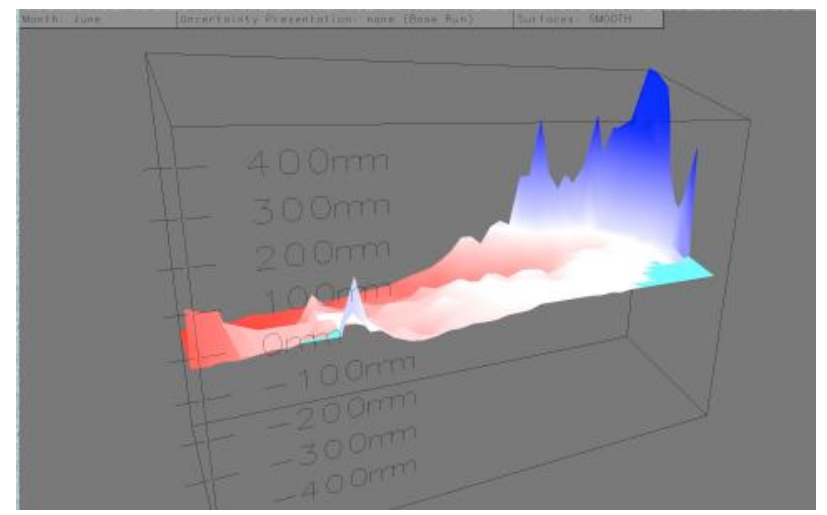

Figure 1: A display of the water budget in a region for the month of June. Surpluses and deficits are blue and red, respectively.

Several types of uncertainties are investigated and illustrated using this model:

- Input data uncertainty. Different global datasets of temperature, precipitation, and soil water holding capacity may lead to alternative estimates of water deficit and surplus, and thus yield a measure of uncertainty.

- $\quad$ Sub-model uncertainty. The overall water balance model includes several important sub-models. We use various Potential Evapotranspiration sub-models to illustrate how different sub-model choices can affect the outcome of the overall model, and hence introduce uncertainty.

- $\quad$ Climate change scenario uncertainty. Ultimately we are interested in the effect of global climate change on water surpluses and deficits. Several different GCM simulations have been developed for global warming [10]. These simulations do not always agree; their differences give us yet another type of uncertainty.
Users can mix and match the above databases and models to see how different sources of uncertainty combine to affect simulation results.

\section{PREVIOUS WORK}

A number of uncertainty display schemes have been reported, and a taxonomy of such schemes has been proposed [7, 13]. Intrinsic schemes modify the color or appearance of an object to indicate the level of certainty in the data. Pang, et. al. [21], for example, have experimented with modifying the diffuse and specular characteristics of objects to make results with high uncertainty appear dull while those with low uncertainty are very shiny. They have also modified surface geometry as a way to show uncertainty in surface fitting.

Wittenbrink, et. al. [28] developed intrinsic schemes applicable to vector fields. Their approach modifies the size, shape, and style of the vectors to visualize uncertainty in both magnitude and direction.

Extrinsic schemes add objects to the display to indicate levels of uncertainty. Cedilnik and Rheingans, for example, add procedurally generated annotation grids which are distorted according to the level of uncertainty and then overlaid on the model being viewed [3]. They found that this highlighted uncertainty without being overly distracting.

Davis and Keller [4] investigate both the use of static uncertainty visualizations like those discussed above as well as animation. As we do here, they focus on how one can present effective displays of uncertainty when there are multiple sources of this uncertainty.

\section{THE WATER BALANCE MODEL}

The regional water balance model is based on the Thornthwaite-Mather approach [15, 24, 25, 26]. Our implementation of the model is similar to that used by Willmott et al. [25].

The overall water balance is based on the following governing equation:

$$
\frac{\partial w}{\partial t}=P_{r} \square E_{a} \square S
$$

where $w$ represents soil water content $\left(0 \prod w \sqcap w^{*} ; w^{*}\right.$ describes soil water holding capacity), $P_{r}$ is the rainfall rate, $E_{a}$ is the actual evapotranspiration rate, and $S$ is the water surplus of a location. $E_{a}$ is calculated as follows:

$$
E_{a}=\begin{array}{cc}
P_{r}+\square\left(w, w^{*}\right)\left[E_{p} \square P_{r}\right] & P_{r}<E_{p} \\
E_{p} & \text { otherwise }
\end{array}
$$

where $E_{p}$ is potential evapotranspiration and $\square\left(w, w^{*}\right)$ is a soil moisture retention function dependent on the ratio of $w$ and $w^{*}$. 
Potential evapotranspiration (PE) is the amount of moisture that would be lost to evaporation and plant transpiration, if it were available. In our system, users are given several alternative methods for calculating PE. One method was developed by Thornthwaite [24] and used by Willmott et al [25] and is based on temperature and latitude. A second PE method was developed by Hamon [8] and uses temperature, daylength and saturation vapor density. Once a method has been chosen to compute PE, we calculate moisture deficit $D=E_{p}-E_{a}$.

Our model simulates a two layer soil system. The top layer accounts for $30 \%$ of the total water holding capacity and will release water freely for evapotranspiration purposes. The lower layer contains $70 \%$ of total soil water holding capacity and will make water available at a linearly decreasing rate until all the water is used up.

Model input requirements include monthly average temperature and precipitation estimates, a soil water holding capacity value $\left(w^{*}\right)$ and the latitude of the location. The model will be run under several different scenarios to represent control and degraded soil conditions which are based on changed soil water holding capacities. Results will present changes in mean annual water surplus $(S)$, deficit $(D)$ and actual evapotranspiration $\left(E_{a}\right)$ conditions.

\section{INPUT DATA}

Basic input data to the current version of our water balance model are temperature, precipitation, and soil water holding capacity. In the context of uncertainty, the source of the input data is critical because the reliability of such data varies considerably on a global scale. In the case of temperature and precipitation, variation in reliability is due to two factors: (1) the weather stations included in different data sets have different criteria for the periods of observation, and (2) the method used to interpolate the raw data to grid points affects the simulation results. In the case of soil water holding capacity, there is a significant uncertainty associated with difficulties in obtaining appropriate averages, and many people still prefer using a fixed value over use of existing databases which are usually derived from less than reliable global data sources.

In the current version of our system, we employ three temperature data sets (two from Legates \& Willmott [11, 27] and one from the Climate Research Unit (CRU) at the University of East Anglia [18]). Similarly, we use three temperature data sets from the same sources $[12,18]$. The only actual soil data set we use is a fairly recently released one [5].

\section{CREATING VISUALIZATIONS}

On startup, our system presents a visual programming model that allows users to select particular gridded temperature, precipitation, and soil datasets (Figure 2). It also permits a choice of potential evapotranspiration (PE) models to be used with the selected temperature and precipitation data. The three gridded datasets along with the output of the Potential Evapotranspiration model are then fed into our Water Balance Model which computes the water budget on the same $1 / 2$ degree by $1 / 2$ degree grid.

Finally the user indicates the region of the world in which they are interested by dragging a rectangle over the area on a world map (Figure 3). The relevant portions of the specified input data sets are then extracted, and the water balance model is computed for that region of the world.

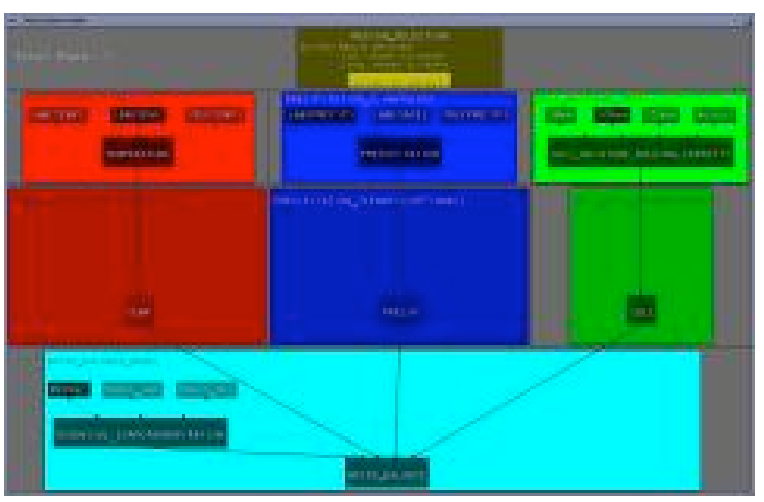

Figure 2: The Visual Programming window

The output from a given run is another gridded data set: the water surplus/deficit at each grid point in the selected region. Since the data is modeled at grid points, the obvious display is a stepped surface (or prism map), in which each cell is projected above or below a base value of 0 (Figure 4). We utilize a redundant color symbolization (blue for surpluses and red for deficits) to enhance the visualization. One can also obtain a continuous representation by determining values at the corners of the cells using a neighbor averaging scheme, but this display often imparts a false sense of how the data changes throughout a cell.

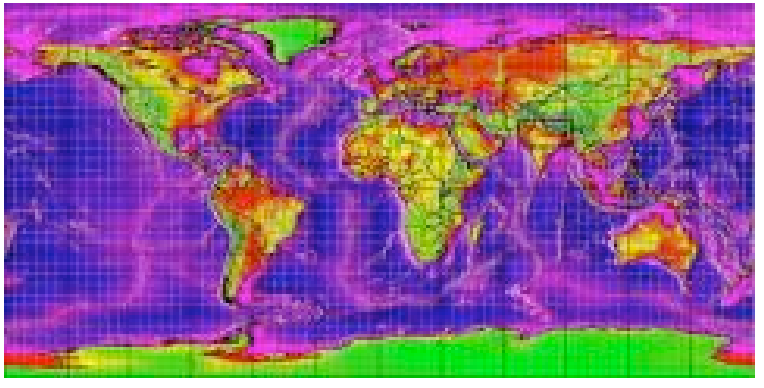

Figure 3: Selecting a region

Once the basic water budget surface is created, users can look for reasons why a particular region has surpluses or deficits. By selecting a point on the surface, for example, we display such information as the monthly precipitation and temperature data for the grid cell associated with that point. Such information may also be useful in assisting the user to isolate errors in the data that might otherwise go unnoticed. 


\section{DEPICTING UNCERTAINTY}

The previous sections focused on how the raw data sets are preprocessed and analyzed to produce visualizations of the general Water Balance Model in a region. As implied throughout the discussion, however, the visualizations really represent guesses based on particular choices of input data sets, the selected PE submodel, and the various climate change scenarios. It is of central importance that our visualization schemes make clear where the results are uncertain, what the magnitude of the uncertainty is, and to what it can be attributed.

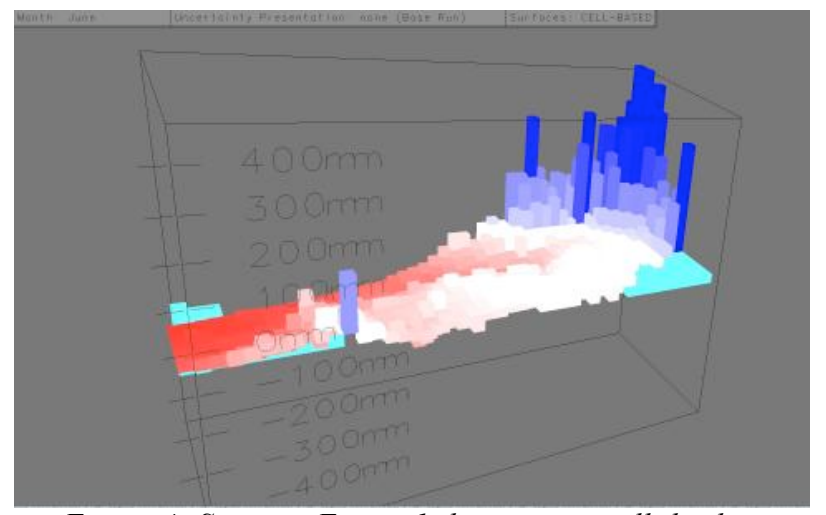

Figure 4: Same as Figure 1, but using a cell display.

First, let us formally define what we mean by error and uncertainty in the context of this work.

Definition: Error is defined as the difference between a recorded value and its actual value. For example, a temperature reading may be incorrectly recorded. Error can be defined only for points in the dataset.

Aside from occasionally being able to spot errors in data from visualizations as suggested at the end of the previous section, our system makes no attempt to locate, visualize, or correct for errors.

Visualizations are constructed by gridding the raw data and feeding the resulting gridded data into various computer realizations of mathematical models of natural phenomena. Uncertainty arises from the assumptions made in the gridding and modeling processes.

Definition: Uncertainty is an inverse measure of the confidence we have in a quantity of interest at some location in our visualization. Uncertainty is defined for all points throughout a displayed region.

As a first step towards computing a measure for and visualizing uncertainty, we recall that uncertainty enters the visualizations in at least the following ways:

- Some of the original data sets have missing data values in some locations and/or times. These missing data values obviously impact the reliability of any interpolation scheme of which they would be a part.

- The original raw data sets vary in quality throughout regions of the world and various time periods. Some are better than others for a specific area of the world or a given period of time.

- The quality of the generated gridded representations depends on the interpolation scheme used, especially in regions distant from actual data gathering stations.

- Once an interpolated representation is generated for each of the required input types (temperature, precipitation, and soil moisture holding capacities), the data are processed by the relevant models: Potential Evapotranspiration and, finally, the Water Balance Model itself. Obviously the accuracy of these models must also be taken into account when making decisions based on visualizations produced.

Our overall goal is to utilize the data sets to feed our models and produce visualizations that not only illustrate resulting estimates regarding the nature of the water budget in a region, but also convey associated uncertainty. For example, we want to not only produce a base prediction for a given region, but also display some indication of the uncertainty by illustrating bounds about this reference surface within which we expect the actual result to lie. These bounds are generated by varying one or more of the quantities identified above as contributors to uncertainty. For example, we could create a surface representing a reference result by selecting the $\mathrm{L} \& \mathrm{~W}$ base temperature climatology, the L\&W base precipitation corrected climatology, and the Dunne and Willmott soil water capacity data. We might then wish to compare this reference result with that obtained from using other choices for temperature and precipitation climatologies.

The basic approach to uncertainty visualization begins with computation of a reference result surface. By varying our choice of raw data sets, gridding schemes, and so forth, we generate several other surface representations of the Water Budget throughout the region. From these other representations, we can compute minimum and maximum values at gridded surface locations and other statistical quantities. Several possibilities exist for using these data to visualize uncertainty. For example, the minimum and maximum surfaces can be used as follows:

- The simplest approach is to draw minimum and maximum surface representations in a color visually distinct from that used for the reference surface. Large gaps between the surfaces highlight areas of high uncertainty.

- When working with large areas, this simple approach oftentimes makes it difficult to isolate internal areas of uncertainty. Therefore, we have developed several alternatives such as displaying bars at the grid locations whose lengths are determined by the minimum and maximum values.

- $\quad$ Recalling that the raw data we are analyzing consist of temperature, precipitation, and soil data, and that we require an intermediate potential evapotranspiration model, we note that there are uncertainties separably 
assignable to each of these four quantities. We can use a variation of the bar display method just discussed to display the uncertainty associated individually with each of these uncertainty sources at each grid location (Figure 5a). This approach is similar to an approach that Onstad et al. [20] utilized to display multivariate data.

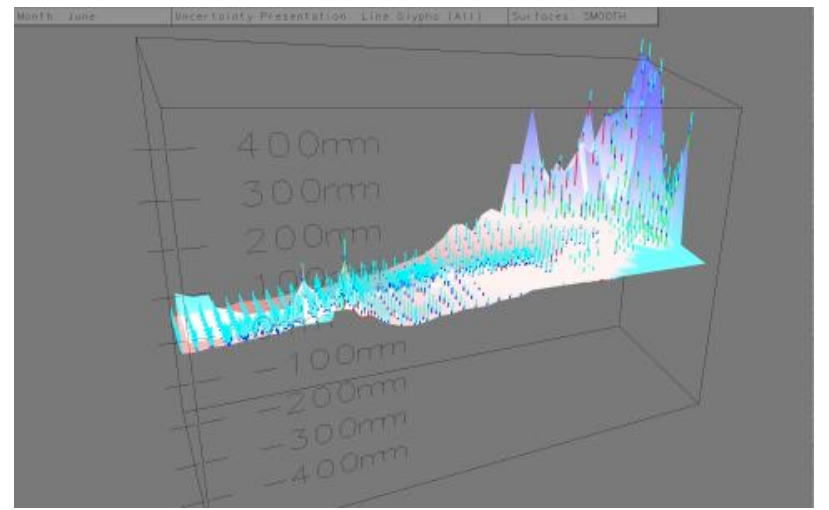

Figure 5(a): A display of four uncertainty bars (red: temperature; blue: precipitation; green: soil; cyan: PE)

- This "four bar" approach can result in displays that are too visually cluttered for large areas. Two possible solutions we have developed are:

○ Show bars for one uncertainty source at a time (Figure 5b).

○ Use a completely different encoding based on mapping the uncertainty associated with temperature, precipitation, and soil moisture holding capacity to, respectively, red, blue, and green colors. By employing a color triangle as a "key", one can see based on color where the various constituent types of uncertainty lie. Surface areas that are black indicate areas of low uncertainty (Figure 6).

\section{SCENARIO BUILDERS}

In addition to selecting a reference surface based on the above datasets, users of our visualization system can also run several specific climate change scenarios. These scenarios involve manipulating the base temperature and precipitation climatologies described above using external data sources that reflect a simulated climate change. The climate change simulations produce temperature and precipitation delta values which are added to the base temperature and precipitation climatologies. The National Center for Atmospheric Research (NCAR), the Hadley Center (UK), and Geophysical Fluid Dynamics Laboratory (GFDL) GCMs were selected for this study, using data obtained from the IPCC Data Distribution Center [10].

The GCM simulations are performed on a much coarser grid than is used by our climatology datasets. We allow the user to select one of two methods for down-scaling the results to the finer $1 / 2$ by $1 / 2$ degree climatology data resolution: a) Direct modification of the $1 / 2$ by $1 / 2$ degree climatology grid cell value based on the predicted change in the overlying GCM grid cell. The advantage of this method is that it conserves the total observed change within each GCM grid cell. Visually, however, this can potentially lead to discontinuities in the data fields.

b) Inverse distance interpolation to the $1 / 2$ by $1 / 2$ degree grid cell value from the nearest neighboring GCM grid cell values. This approach does not conserve the total observed change in each GCM grid cell, but provides a smoother climate change data field.

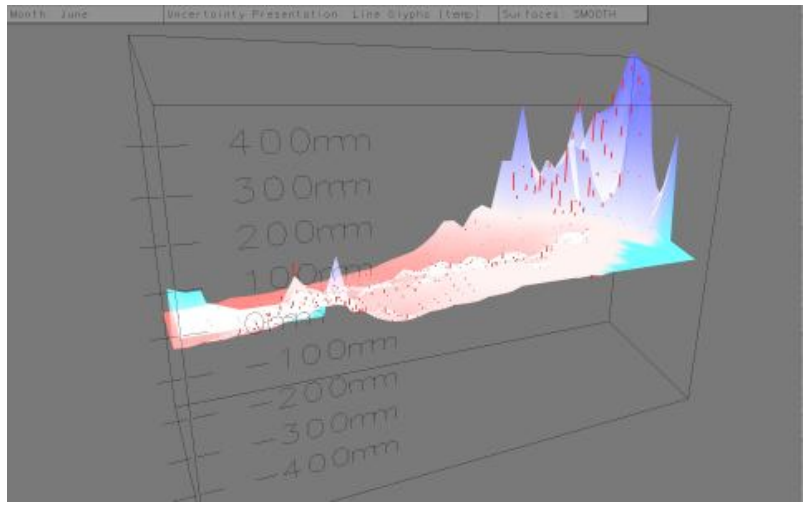

\section{Figure 5(b): Displaying only temperature uncertainty bars.}

Once the downscaling method has been selected, the user can select a GCM and view the resulting climate change prediction. If more than one GCM simulation is selected, any of a variety of climate change uncertainty presentation schemes can be used to explore the areas of variation as well as the source of the uncertainty.

\section{USABILITY}

Although many interesting visualization systems have been developed in environmental modeling and elsewhere, few of them have been evaluated from a user standpoint. Such an evaluation can be accomplished using the principles of usability engineering (e.g., [16, 19]), which actively involves users throughout the lifecycle of software design, development, and deployment. In the field of cartography, the notion of usability engineering has generally involved a limited portion of the software lifecycle, a limited range of user testing methodologies, and has simply been called "user testing" (e.g., [9, 14, 17]). More recently, cartographers have recognized the importance of usability engineering [23], and studies are now being undertaken that consider associated guidelines (e.g., $[1,6]$ ).

We tested our system with three different types of users at three different stages of development. Our first wave of testing involved domain experts, individuals who were familiar with the science and who could tell us major functional capabilities that needed to be present. Our 
second wave of testing involved usability experts who provided considerable feedback which led to significant changes to the interface and basic interactive gestures. After incorporating changes suggested during these first two testing phases, we brought in a series of decision makers. Included in this group were a state representative and staff members of a U.S. Representative. We met with them individually, interviewed them to learn about how the decision making process worked from their perspective, and then finally posed a problem which related to water resources and crop production strategies.

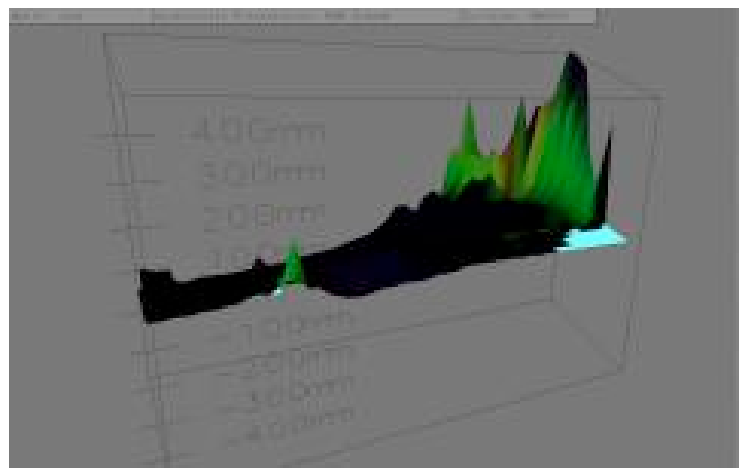

Figure 6(a): Using color mixtures to display multiple sources of uncertainty

Obviously these decision makers did not have adequate knowledge of the science to be able to use the system to answer the question. We have from the start viewed our system as fundamentally collaborative - in this case the collaboration requires a scientist or domain expert to work with the decision maker to work through the science and help the decision maker combine good science with social and political factors to arrive at good public policy. For this wave of testing, we therefore used our own resident climatologist (one of the authors) as the domain expert working with the decision maker. This worked very well.

It is beyond the scope of this paper to discuss the results of this testing in detail, but the feedback we received from the decision makers was especially positive. They felt that they gained considerable new insight into the use of scientific models in general as well as climatology applications and concepts in particular.

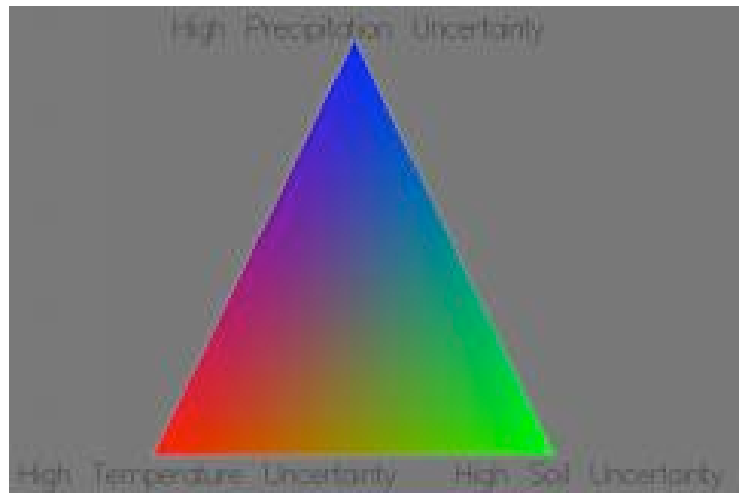

Figure 6(b): The color key used to interpret uncertainty

\section{SUMMARY}

We have described a collaborative visualization system for decision support applications which enables decision makers in collaboration with members of their technical staff and/or scientists to compute and visualize simulated water budgets for any region of the world. Moreover, we provide decision makers with the ability to see the extent to which the results of these models are uncertain, and we provide tools to create and analyze future scenarios describing how potential policy decisions may affect the water budget in some region. We provide tools to visualize the sources of these uncertainties. The uncertainties in the results may arise from our choice of input data (temperature and precipitation climatologies, and soil water holding capacity), our choice of sub-model for Potential Evapotranspiration, our choice of GCM simulations of climate change, or from some combination of these. We have formally evaluated and improved the usability of the system using well-established usability engineering principles.

Future plans for the system include various types of improved interpolation algorithms in both the spatial and temporal domains. We are developing a more sophisticated water budget model which would incorporate effects such as snow melt. Selecting rectangular regions is oftentimes inadequate, hence we are developing more general methods for specifying a region of interest including selections based on country boundaries and watershed regions. Finally, we are pursuing improved uncertainty analysis on several fronts, including better display methods when many sources of uncertainty are present and possible ways to hook the identification of uncertainty to decision making strategies to help a decision maker cope with uncertainty in scientific models.

\section{ACKNOWLEDGMENTS}

The work described here was performed in DesignLab, a multidisciplinary research laboratory at the University of Kansas funded in part by NSF Grant CDA-94-01021. The University of Kansas Center for Research Development Fund, grant 2300502, provided additional support. Special thanks should also go to the many test subjects who traveled to our lab to participate in the evaluation of this software.

\section{REFERENCES}

[1] I. Brewer, The design and implementation of temporal, spatial, and attribute query tools for geovisualization. In: Association of American Geographers Annual Meeting, New York, New York, 2001.

[2] B. Buttenfield, Usability evaluation of digital libraries. Science \& Technology Libraries 17 (3/4): 39-59, 1999.

[3] A. Cedilnik and P. Rheingans, Procedural Annotation of Uncertain Information, Proceedings IEEE Visualization 2000, Salt Lake City, Utah, 2000, pp. 77-84. 
[4] T. J. Davis and C. P. Keller, Modelling and Visualizing Multiple Spatial Uncertainties, Computers and Geosciences, Vol. 23, No. 4, 1997, pp. 397-408.

[5] K. Dunne and C. Wilmott, Global Distribution of plant-extractable water capacity of Soil, International Journal of Climatology, August 1996.

[6] R. M. Edsall, Assessing geovisualization effectiveness: A paradox and an approach. In: Association of American Geographers Annual Meeting, New York, New York, 2001.

[7] N. Gershon, Visualization of an Imperfect World, IEEE Computer Graphics and Applications, July/August, 1998.

[8] W. R. Hamon, Equation for estimating potential evapotranspiration in terms of a temperature function and day length. Methods for estimating evapotranspiration. Irrigation and Drainage Specialty Conference. Amer. Soc. Civil Eng. New York, N.Y., 1966.

[9] M. Harrower, A. MacEachren and A. L. Griffin, Developing a geographic visualization tool to support earth science learning. Cartography and Geographic Information Science 27 (4): 279-293, 2000.

[10] IPCC, http://ipcc-ddc.cru.uea.ac.uk/, 2001.

[11] D. R. Legates and C. J. Willmott, Mean Seasonal and Spatial Variability Global Surface Air Temperature, Theoretical and Applied Climatology, 41, 11-21, 1990.

[12] D. R. Legates and C. J. Willmott Mean Seasonal and Spatial Variability in Gauge-Corrected, Global Precipitation. International Journal of Climatology, 10, 111-127, 1990.

[13] A. MacEachren, Visualizing Uncertain Information, Cartographic Perspective, 13, pages 10-19, 1992.

[14] A. MacEachren, F. P. Boscoe, D. Haug and L. W. Pickle, Geographic visualization: Designing manipulable maps for exploring temporally varying georeferenced statistics. In: Proceedings, Information Visualization '98, Research Triangle Park, North Carolina, IEEE Computer Society, pp. 87-94, 1998.

[15] J. Mather, The Climatic Water Budget in Environmental Analysis, Lexington Books, Lexington, Massachusetts, 1978.

[16] D. J. Mayhew, The Usability Engineering Lifecycle: A Practitioner's Handbook for User Interface Design. San Francisco, California: Morgan Kaufman Publishers, Inc., 1999.
[17] M. Monmonier and M. Gluck. Focus groups for design improvement in dynamic cartography. Cartography and Geographic Information Systems 21 (1): 37-47, 1994.

[18] M. New, M. Hulme, and P. Jones, Representing twentieth-century space-time climate variability. Part I: Development of a 1961-90 mean monthly terrestrial climatology. J. Climate, 12, 829-856, 1999.

[19] J. Nielsen, Usability Engineering. Boston, Massachusetts: Academic Press, Inc., 1993.

[20] D. W. Onstad, J. V. Maddox, D. J. Cox and E. A. Kornkven, Spatial and temporal dynamics of animals and the host-density threshold in epizootiology. $J$. of Invertebrate Pathology 55 (1): 76-84, 1990.

[21] A. Pang C. Wittenbrink, and S. Lodha, Approaches to Uncertainty Visualization, The Visual Computer, Vol. 13, No. 8, November 1997, pp. 370-390.

[22] D. Shepard, A two-dimensional Interpolation function for irregularly-spaced Data. Proceedings, 1968 ACM National Conference, 517-523, 1968.

[23] T. A. Slocum, C. Blok, B. Jiang, A. Koussoulakou, D. R. Montello, S. Fuhrmann and N. R. Hedley, Cognitive and usability issues in geovisualization. Cartography and Geographic Information Science 28 (1): 61-75, 2001.

[24] Thornthwaite, An Approach to a Rational Classification of Climate, Geography Revue, Vol. 38, No. 1, pages 55-94, 1948.

[25] C. J. Willmott, C. M. Rowe, and W. D. Philpot, Small-Scale Climate Maps: A Sensitivity Analysis of Some Common Assumptions Associated with Grid-point Interpolation and Contouring. American Cartographer, 12, 5-16, 1985.

[26] C. J. Willmott and K. Matsuura, Smart Interpolation of Annually Averaged Air Temperature in the United States, J. of App. Meteorology, 34, 2577-2586, 1995.

[27] C. J. Willmott, K. Matsuura, and D.R. Legates, 1998 Global Air Temperature and Precipitation: Regridded Monthly and A $\mathrm{n} \mathrm{n} \mathrm{u} 1 \quad$ C $1 \mathrm{i} \mathrm{m}$ a $\mathrm{t}$ o 1 o $\mathrm{g}$ i e s, http://climate.geog.udel.edu/ climate/html pages/READM E.lw.html

[28] C. M. Wittenbrink, A. T. Pang, and S. K. Lodha, Glyphs for Visualizing Uncertainty in Vector Fields, IEEE transactions on Visualization and Computer Graphics, Vol. 2, No. 3, September 1996, pp. 266-279. 